\title{
Pañetes de tierra estabilizada con nopal
}

\section{Earthen plasters stabilized with prickly pear cactus}

\author{
Luis Fernando Guerrero Baca* y Esmeralda Ávila Boyas**
}

\section{Resumen}

Los recubrimientos de la arquitectura de tierra cumplen diversas funciones entre las que se destacan la protección de las estructuras, la regularización de las superficies para hacerlas más higiénicas (por no tener huecos en los que habite microflora o fauna) y la posibilidad del intercambio de vapor de agua con el aire circundante. Este artículo presenta los avances de una investigación que estudia el posible mejoramiento de pañetes de tierra a partir de la recuperación de tradiciones regionales derivadas del uso del mucílago de la cactácea conocido en México como "nopal" (Opuntia spp.). Los resultados obtenidos hasta el momento muestran un incremento notable en la plasticidad y adherencia de las mezclas, una ralentización del secado que mejora la uniformidad de las superficies, un ahorro en el volumen de agua para mezclado y un retraso en el tiempo de absorción hídrica por capilaridad cercano al 300\%.

Palabras clave: sostenibilidad, tradición constructiva, revoques, aglutinante, mucílagos.

\section{Abstract}

Earthen architecture coatings fulfill with several functions such as protection of the structures, regularization of surfaces to make them more hygienic (for not having gaps in which microflora or fauna inhabit) and the water vapor exchange with the surrounding air. This article presents the advances of a research that studies the possible improvement of mud plasters from the recovery of regional traditions derived from the use of the mucilage of the cactus known in Mexico as "nopal" (Opuntia spp.). Results currently found show a remarkable increase in plasticity and adherence of mixtures, a slowdown in the drying time which improves the uniformity of surfaces, a saving of water for mixing and a delay near to $300 \%$ on moisture absorption.

Keywords: sustainability, traditional construction, plasters, binder, mucilage.

Fecha de recepción: 5 de marzo de 2019 - Fecha de aceptación: 8 de agosto de 2019

* Doctor en Diseño con especialidad en Conservación y restauración del patrimonio construido, Universidad Autónoma Metropolitana, CDMX, México. Correo electrónico: luisfg1960@yahoo.es

** Magister en Arquitectura, Universidad Nacional Autónoma de México. Correo electrónico: arqavilaboyas80@gmail.com 


\section{Introducción}

Entre los componentes más importantes de la arquitectura de tierra se destacan sus pañetes por ser los encargados de mantener el equilibrio en la relación entre los edificios y su entorno. Aunque existen evidencias de estructuras térreas que han subsistido sin revoques en muchos lugares del orbe y a lo largo de diferentes periodos históricos -como sucede con ruinas arqueológicas del medio oriente o del norte de México y del Perú- la mayor parte de las obras antiguas y recientes, ha recibido durante su vida útil revestimientos destinados a protegerlas de las inclemencias climáticas, del ataque de flora o fauna parásita y de afectaciones antrópicas.

Por su condición protectora, los revestimientos de los edificios tienen que ser lo suficientemente tenaces como para resistir acciones mecánicas

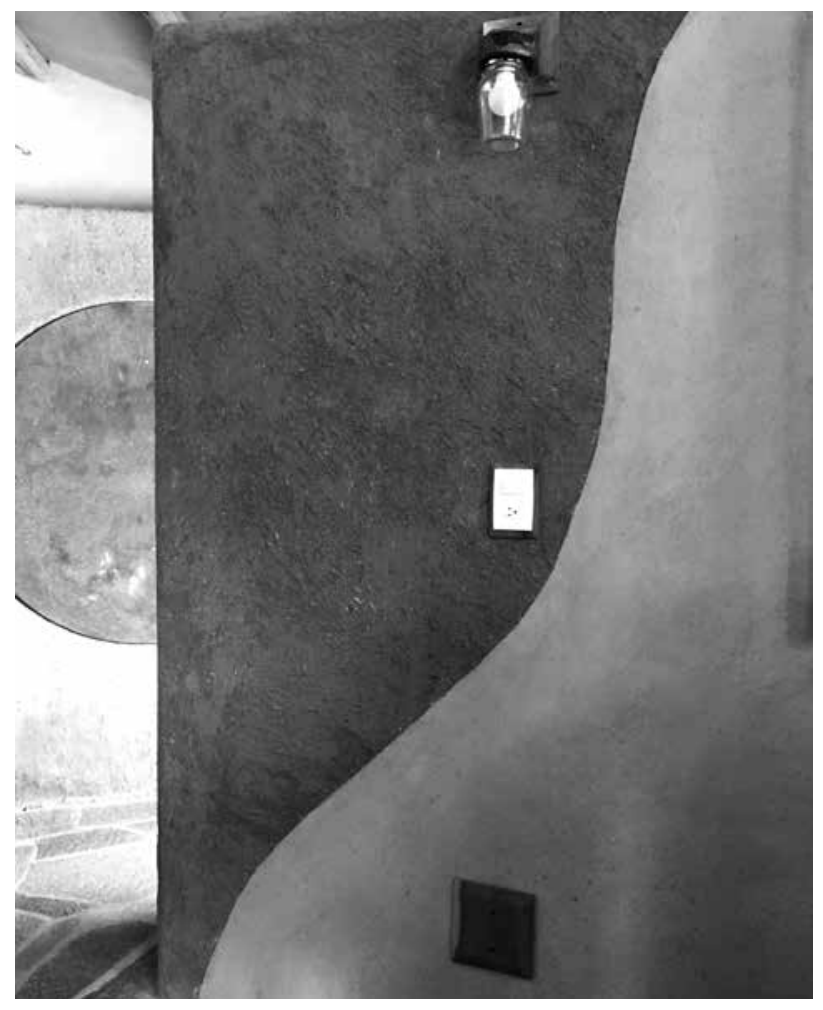

Figura 1. Los revestimientos de tierra brindan condiciones de confort al regular el flujo del vapor de agua. Obra de la Arquitecta Alejandra Caballero, Tlaxcala, México. Fuente: Guerrero (2016). y biológicas, así como el impacto del agua de lluvia. Pero, además, han de tener la capacidad de desarrollar respuestas dúctiles ante sismos y hundimientos diferenciales, y ser permeables al vapor de agua para mantener el comportamiento higrotérmico que siempre se ha valorado en la construcción con tierra (Figura 1).

Estos elementos deben poseer la capacidad de permanecer adheridos a las superficies murales por mucho tiempo con el fin de prolongar su vida útil y extender la periodicidad de las necesarias labores de mantenimiento preventivo (Lozano, 2008).

Para cumplir estos requerimientos, a lo largo de la historia se han utilizado diferentes tipos de substancias estabilizantes que, además de propiciar la adhesividad de los pañetes a los sustratos térreos, les confieran la consistencia necesaria para resistir la erosión y el efecto del agua en estado líquido. Sin embargo, como consecuencia de las propiedades higroscópicas de la tierra, no es conveniente que los repellos sean totalmente impermeables porque se sabe que esta es una de las principales causas de su deterioro, como lamentablemente ha sucedido con los enlucidos de cemento y las pinturas plásticas (Guerrero, 2016).

La incorporación de substancias impermeables ha demostrado ser muy nociva para las obras históricas y tradicionales realizadas con materiales porosos como la piedra, el ladrillo, la cal, la madera y, sobre todo, la tierra. Su presencia provoca la migración de sales solubles que cristalizan en las superficies al contacto con el aire, las cuales finalmente caen por su propio peso, arrastrando partículas de las estructuras patrimoniales (Warren, 1999).

Este efecto dañino se presenta en un elevado número de edificios de tierra que han sido supuestamente "protegidos" mediante la colocación de pañetes de cemento los cuales limitan el adecuado intercambio cíclico del aire y vapor de agua con el medio ambiente. Entonces, la humedad se acumula en el interior de los muros y progresivamente se van disgregando tanto los recubrimientos como los componentes constructivos del núcleo (Guerrero, Correia y Guillaud, 2012). 


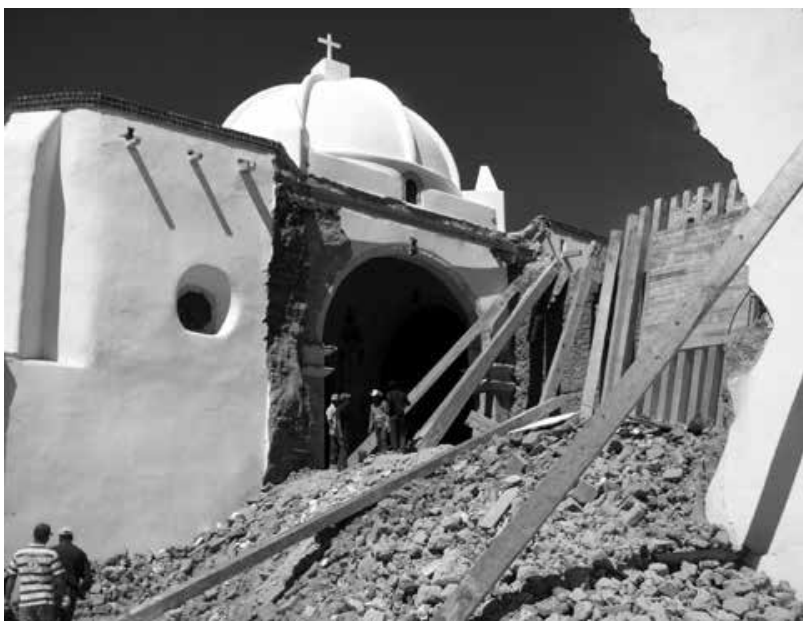

Figura 2. El muro sur del templo de Satevó, Chihuahua, México, construido con adobe, se derrumbó intempestivamente a consecuencia del uso de revoques de cemento. Fuente: Guerrero (2016).

En casos todavía más graves, al no conseguirse una apropiada adherencia de los repellos de cemento, éstos se han aplicado sobre mallas metálicas previamente clavadas a las superficies. Además de la afectación provocada a los inmuebles por las múltiples perforaciones, el problema del encapsulamiento de la humedad se agrava al disfrazarse. Las capas de cemento no permiten ver los procesos de desintegración interna de los sistemas y después de algún tiempo los componentes constructivos de tierra pierden su capacidad de carga y, en casos extremos, puede sobrevenir su colapso (Figura 2).

En el polo opuesto, existen diferentes recursos de origen tradicional que han sido muy exitosos en el mejoramiento de mezclas aplicadas a pañetes. Para el caso de México destaca el jugo de la planta conocida como nopal (Opuntia spp.), que se ha usado desde la época prehispánica (Pérez, 2009). Se sabe que al adicionarse este líquido viscoso como parte del agua de mezclado, se incrementa la plasticidad y adherencia de los morteros y recubrimientos de cal (Kita y Daneels, 2015). La substancia activa del jugo de nopal posee mucílago $y$ pectina, $y$ ha sido documentada su presencia en análisis químicos de muros y pisos realizados hace más de mil años en la zona arqueológica de Teotihuacán (Cruz, 2013).
Desde tiempos remotos y todavía en la actualidad, en diversos sitios tradicionales de México se añade "baba de nopal" a las pinturas y lechadas. Asimismo, en el campo de la conservación y restauración de monumentos, se suele recomendar y aplicar en la mayor parte de las intervenciones en las que se incluyen mezclas de cal.

Recientemente se ha demostrado que además de ayudar a la adherencia de las mezclas por su consistencia viscosa, el mucílago tiene la capacidad de propiciar y acelerar el desarrollo de cristales de hidróxido de calcio de pequeñas dimensiones, con lo que se optimiza su rendimiento como parte de morteros de pega y revoque (Pérez, Guerrero, González y Prado, 2017).

Sin embargo, aunque el uso de jugo de nopal en pañetes de tierra sea un procedimiento tradicional del centro y norte de México, ha sido poco estudiado de manera sistemática. Es por esto que surgen diversas interrogantes acerca del papel que puede jugar como parte de los procesos constructivos. Por este motivo se planteó una serie de ensayos tendientes a verificar si realmente el mucílago afecta el comportamiento de la tierra como parte de las mezclas utilizadas para pañetes, si mejora su consistencia, adherencia y trabajabilidad, y si una vez que endurece se modifica su capacidad de carga, absorción capilar y, sobre todo, su resistencia al impacto de la lluvia.

Interesa también verificar si dadas las capacidades higroscópicas del mucílago, su empleo permite conseguir una plasticidad adecuada utilizando un menor volumen de agua de mezclado. De este modo se podría reducir su consumo con lo que se tendría adicionalmente un manejo más sostenible de los recursos hídricos.

Por otra parte, se intenta documentar si el empleo de mucílago de nopal propicia un secado más lento y estable de la mezcla, pues se sabe que un factor crucial en el diseño de estructuras de tierra está asociado con su velocidad de endurecimiento (Pérez, 2009). Si el bahareque, adobes, morteros o pañetes se secan violentamente se presentan agrietamientos superficiales que reducen la resis- 
tencia de los elementos constructivos. En cambio, una pérdida gradual del agua de mezclado permite un mejor acomodo de las micelas que conforman las arcillas traduciéndose en componentes más homogéneos y duraderos.

Finalmente, se busca evaluar la posibilidad de aplicar pañetes de tierra no solamente sobre muros construidos con este mismo material, sino también sobre superficies realizadas con sistemas constructivos industrializados como los que conforman la mayor parte del medio urbano actual. El empleo de acabados de tierra sobre muros de ladrillo o bloques de cemento que normalmente se dejan sin repello en las viviendas de escasos recursos, además de mejorarlos estéticamente, permitiría aportar cualidades de higiene y confort al interior a los espacios habitables (Figura 3).

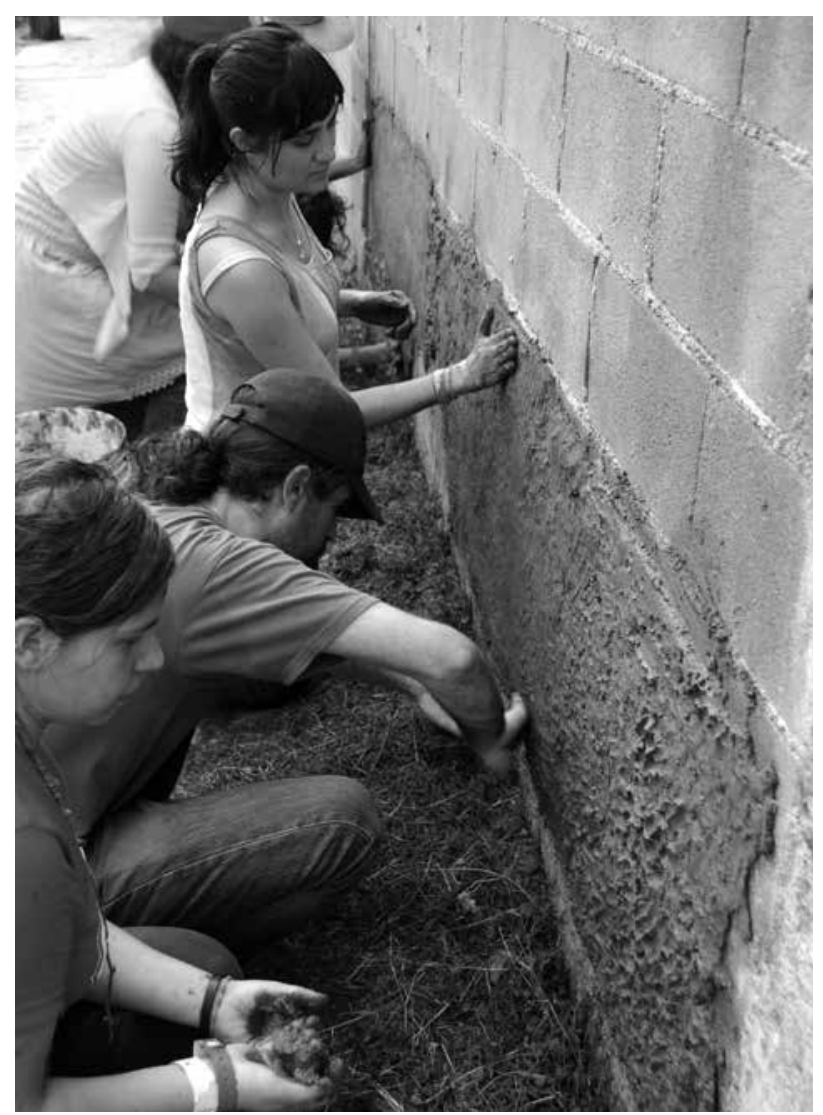

Figura 3. Aplicación de pañetes de tierra con mucílago de nopal sobre un muro de bloques de cemento. Taller comunitario realizado en Santiago, Nuevo León, México. Fuente: Guerrero (2015).
El incremento en las cualidades de los pañetes hechos con tierra será muy positivo tanto para la conservación de estructuras patrimoniales y vernáculas, como en el campo de la construcción contemporánea de tierra y como apoyo a la vivienda precaria. De esta manera se podría abatir el empleo de substancias artificiales cuya fabricación y aplicación generan un elevado impacto ambiental (Guerrero, 2015).

\section{Metodología}

El presente texto muestra los avances de una serie de experimentos dirigidos hacia la verificación de la viabilidad de emplear técnicas históricas y tradicionales para elaborar mezclas de tierra estabilizada con mucílago de Opuntia ficus. Se espera obtener revoques que resulten sostenibles desde la perspectiva económica, social y ecológica, al ser aplicados tanto sobre muros de tierra como de otros materiales constructivos.

Se detallan algunos análisis comparativos de mezclas de un mismo tipo de tierra adicionada por una parte sólo con agua, y por otra, con una cantidad regulada de mucílago. Se realizaron pruebas de cohesividad, retracción superficial, retracción volumétrica, absorción capilar, tiempo de secado, adherencia sobre diferentes sustratos, resistencia a la compresión y efecto de la lluvia.

La tierra que se utilizó para la elaboración de las muestras en esta investigación fue colectada de la zona de Tepecoacuilco, Guerrero, al sur de la República Mexicana. Los datos proporcionados por el laboratorio Geotecnia y Suelos indicaron que contenía $17 \%$ de arena y $83 \%$ de finos, un Límite Líquido de 64,3 y un Límite Plástico de 26,3, por lo que se concluyó que se trata de una "Arcilla Inorgánica Limoarenosa" correspondiente al tipo "CH" del Sistema Unificado de Clasificación de Suelos (SUCS) (Juárez y Rico, 2010).

La elección de un material altamente plástico se derivó de la intención de poder compensarlo mediante el agregado de volúmenes conocidos de arena comercial de mina y de este modo contar 
con una materia prima regularizada para todas las pruebas.

Las actividades partieron de la identificación de la cantidad de arena que se requeriría para compensar la fuerza de la tierra arcillosa. Ambos agregados se tamizaron por la malla \#10 (2mm). Se realizaron diferentes mezclas variando la dosificación relativa del volumen de arena y con cada una se procedió a hacer pañetes de $20 \times 20 \mathrm{~cm}$ de área y $2 \mathrm{~mm}$ de espesor, aplicados sobre una superficie lisa expuesta al sol. Una vez que se secaron las muestras se verificó el patrón de agrietamiento que presentaban y se concluyó que la dosificación que menos se había fisurado, pero que conservaba todavía una buena consistencia sin disgregarse, era la que contenía 1 volumen de tierra por 1,5 volúmenes de arena. Se mezcló en seco una cantidad suficiente de ambos componentes ya tamizados y esa fue la materia prima para todas las pruebas.

Esta mezcla se envió nuevamente al laboratorio y se comprobó que tras la compensación había alcanzado un volumen de $81 \%$ de arena y $19 \%$ de finos, de manera que su Límite Líquido ahora era de 23,4 y su Límite Plástico de 15,0, habiéndose "transformado" en una "Arena Arcillolimosa" correspondiente al tipo "SC" del Sistema Unificado de Clasificación de Suelos (SUCS) (Juárez y Rico, 2010).

Para dosificar la solución de mucílago en agua se diseñó una prueba que consiste en agregar un volumen conocido de pulpa de nopal extraída en seco (Pérez et al., 2017) en un balde de agua, dejando reposar la solución por un periodo de 48 horas. Pasado este tiempo, se toma un pequeño volumen de la solución con un recipiente pequeño y se vierte sobre otro elevando el punto de caída a fin de poder medir la longitud del chorro continuo del material viscoso. Si el hilo se interrumpe cayendo en forma de gotas a poca altura significa que la solución requiere mayor cantidad de mucílago concentrado. En cambio, si el hilo no se interrumpe al levantar el punto de vertido, quiere decir que es posible agregarle más agua. La solución se considera adecuada cuando la longitud del hilo es continua desde la altura de vertido de un metro.
Esta estrategia de evaluación es muy útil debido a que se sabe que las características de los extractos varían notablemente en función de la especie de nopal, su grado de madurez, su momento de corte y el proceso de extracción, entre muchos otros factores (Torres et al., 2015). Por ello, al evaluar el comportamiento esperado de la mezcla con agua a partir de una forma fácilmente observable de su viscosidad por decantación, se abre la posibilidad de utilizar cualquier tipo de nopales sin importar los factores antes descritos.

En este sentido conviene mencionar que las diversas variedades de cactáceas del género Opuntia tienen presencia en muchos lugares del mundo y generalmente se desconoce su potencial de uso en la edificación. La recuperación de estos saberes asociados a las culturas constructivas de tierra (Figura 4) permitirá su cultivo y aprovechamiento sostenible en regiones donde incluso se considera una especie de plaga.

La investigación se realizó tanto en el Laboratorio de Materiales Tradicionales de la Escuela Nacional de Conservación Restauración y Museografía (ENCRyM-INAH) como en la Universidad Autónoma Metropolitana Xochimilco, ambas en la Ciudad de México. Los estudios han tenido diferentes etapas pero se sistematizaron durante los últimos dos años.

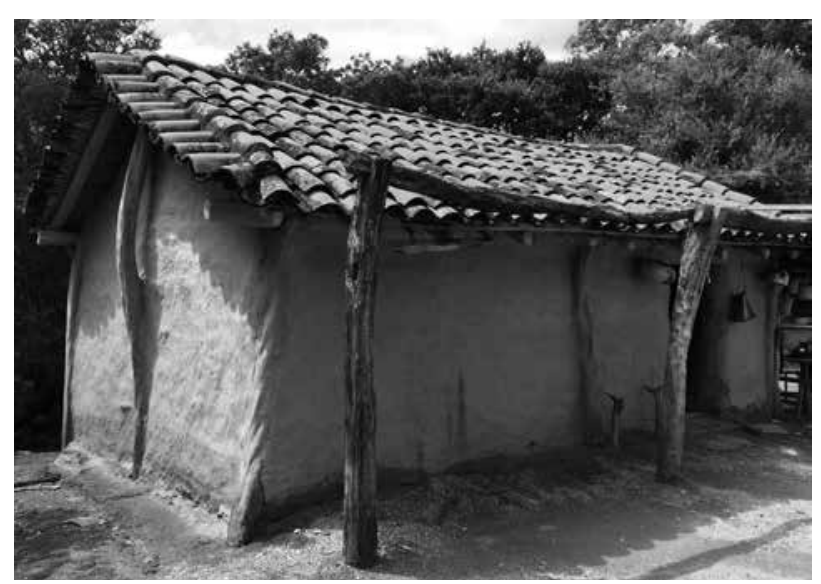

Figura 4. Las comunidades rurales conservan sus espacios habitables mediante labores periódicas de revestimientos con tierra, Ixtepec, México. Fuente: Guerrero (2015). 
La primera evaluación comparativa que se llevó a efecto buscaba medir la cohesividad. A falta de una norma estandarizada para el caso de la tierra se tomó como referencia el procedimiento sugerido por Minke (2005) con algunas variaciones dimensionales. Se realizaron simultáneamente mezclas de tierra con agua y tierra con mucílago hasta que cada una de ellas adquiriera la consistencia necesaria como para hacer una esfera al amasarla con la mano. Una vez que las esferas alcanzaron la plasticidad necesaria, se colocaron sobre una superficie lisa y seca para realizar unas barras cilíndricas de $20 \mathrm{~cm}$ de largo por $1.5 \mathrm{~cm}$ de diámetro. Inmediatamente cada barra se colocó sobre una hoja de papel y se puso en la orilla de una mesa para ayudar a dejarla colgar hasta el punto en que se rompiera. Cada fracción rota se recibía cuidadosamente para evitar que cayera al suelo y se pudiera deformar porque el dato buscado era la longitud a la que se rompía.

Durante el desarrollo de la prueba se pudo observar que se requirió menor cantidad de solución de mucílago para alcanzar la plasticidad necesaria en comparación con las esferas amasadas solamente con agua. Entonces se repitió el procedimiento midiendo con más precisión la cantidad de agua requerida en ambos casos y se registraron los resultados.

A continuación se utilizaron sendas mezclas para hacer unas placas de $7 \times 7 \mathrm{~cm}$ de superficie y $5 \mathrm{~mm}$ de espesor en unos moldes de madera. Se pesaron en una báscula de precisión y se pusieron a secar al sol registrándose su peso cada media hora hasta que en tres mediciones continuas este no variara. Se tomó nota del tiempo final que era el dato central del experimento.

El cuarto estudio se dirigió hacia la evaluación comparativa de pruebas de compresión simple para la que se realizaron cubos estándares de $5 \times 5 \times 5 \mathrm{~cm}$ correspondientes a ambas series: con tierra y agua, así como con tierra y mucílago disuelto. Todas las probetas se secaron por completo a temperatura y humedad ambiental durante un mes. Fueron empleadas como referencia las normas mexicanas
NMX-C-036 -ONNCCE (2004) y ASTM C-39 (2006), con algunas adecuaciones derivadas de la forma y dimensiones de las probetas (RamírezArellanes, et al, 2012). Se probaron nueve probetas de cada dosificación en la prensa manual ELVEC 657-2 digital localizada en el Laboratorio de Materiales de la Universidad Autónoma Metropolitana. Unidad Xochimilco.

En quinto lugar, otras series de cubos de las mismas dimensiones se emplearon para realizar las pruebas de absorción capilar en una probeta de cada dosificación. Como actualmente en México no existen estándares que permitan determinar esta variable en componentes de tierra, se tomó como referencia la norma italiana Normal 11-85 (CNR-ICR, 1985).

En ese ensayo, cada muestra se coloca sobre una tela saturada de agua en una charola durante periodos de 30 segundos y después se pesa en una báscula de precisión. Se repite el proceso hasta que la muestra comience a tener pérdida del material por desmoronamiento y por lo tanto los resultados se empiecen a volver inconsistentes.

Para la sexta y última evaluación se realizaron pañetes de 20 x $20 \mathrm{~cm}$ de superficie y $2 \mathrm{~mm}$ de espesor en diferentes materiales constructivos. Se aplicó un pañete de cada dosificación sobre madera, adobes, ladrillos, bloques de cemento e incluso sobre un muro de concreto con un terminado notablemente liso.

En todos los casos se colocaban por pares a fin de comparar los repellos que habían sido mezclados con agua y los de mucílago. Las muestras se dejaron a la intemperie para evaluar su comportamiento ante la lluvia directa.

\section{Resultados}

La prueba de cohesividad se repitió seis veces con ambas mezclas para evitar posibles fallas derivadas del amasado o la ejecución de las barras (Figura 5). Se pudo observar que las fracciones correspondientes a las barras realizadas con agua se rompieron en promedio a los $5,8 \mathrm{~cm}$. En cambio 


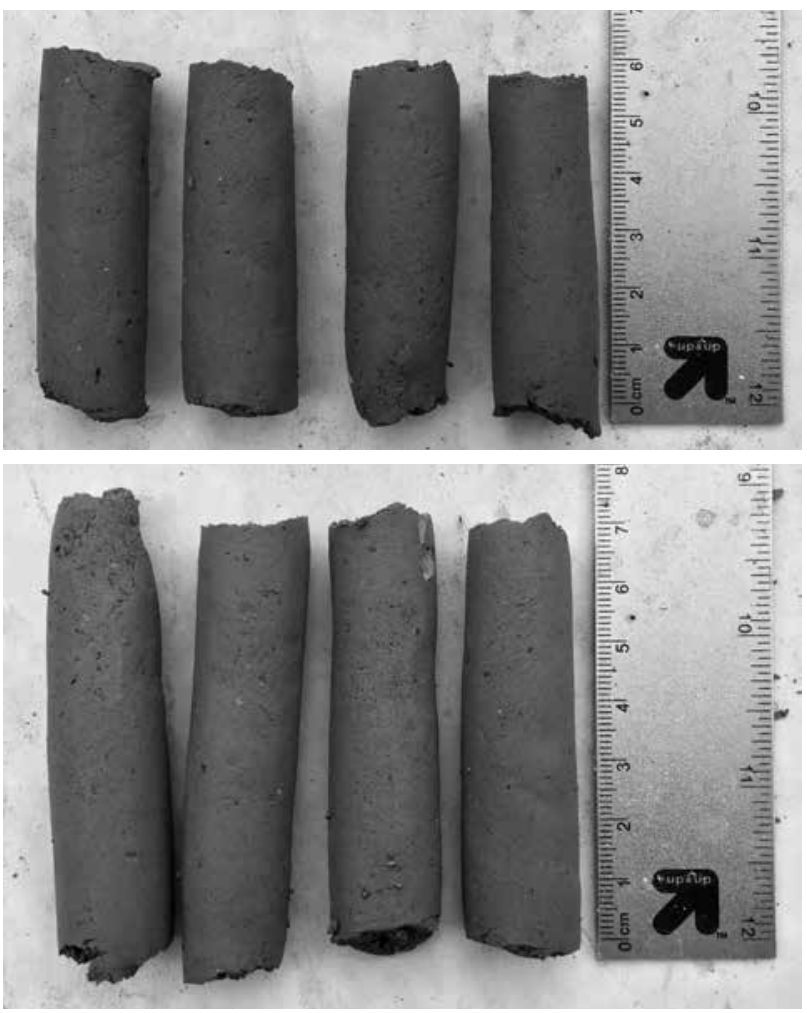

Figura 5. Arriba se observan las fracciones de la tierra amasada con agua y abajo las de mucílago disuelto. Fuente: Guerrero (2015).

las barras que tenían mucílago como líquido de mezclado se rompieron en promedio a $\operatorname{los} 7,1 \mathrm{~cm}$.

Con respecto a la cantidad de agua requerida para la obtención de un mortero adecuadamente plástico y que permitiera un amasado uniforme, se observó que la mezcla de tierra-arena-agua requirió $175 \mathrm{ml}$ mientras que la de tierra-arenamucílago sólo $135 \mathrm{ml}$.

En tercer lugar, la evaluación de la pérdida de peso de las series de placas delgadas expuestas al secado directo al sol mostró que las probetas de agua se secaron por completo en 6 horas con cuarenta minutos mientras que las de mucílago lo hicieron en 9 horas con doce minutos (Figura 6). Esto representa un retardo en el tiempo de secado del $38 \%$.

En lo que se refiere a las pruebas de compresión de las probetas cúbicas, se sometieron nueve muestras por cada mezcla a carga axial en la prensa y el promedio de los resultados para la mezcla de tierra

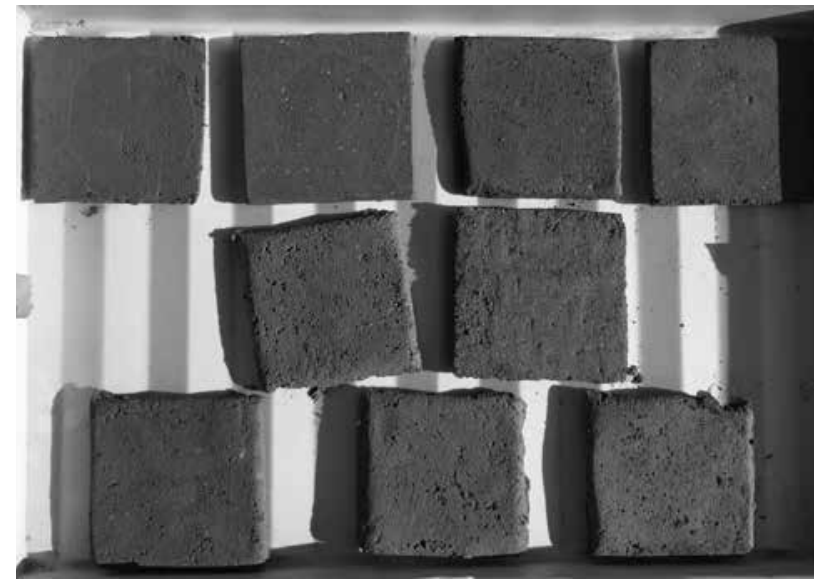

Figura 6. A las cinco horas del experimento, las placas que aparecen en la parte inferior de la foto -y que fueron amasadas con agua- ya estaban prácticamente secas mientras que las cuatro superiores todavía se notan obscuras por su estado húmedo. Fuente: Guerrero (2015).

con mucílago fue de 5,4 Mpa mientras que para los de tierra con agua fue de 7,0 Mpa. Esto significa que el uso del mucílago redujo la capacidad de carga de las muestras en un 29,62\%.

Luego, la prueba de absorción capilar se llevó a cabo registrando y tabulando el incremento en el peso de las probetas sumergidas en agua en periodos intermitentes de treinta segundos durante un lapso total de ocho minutos. La cantidad de agua que absorbieron las muestras se graficó para comparar la diferencia de pesos en cada momento.

Para determinar el coeficiente de absorción (Abs) se utilizó la siguiente fórmula:

$A b s(\%)=\frac{P s-P d}{P d} \times 100$

Dónde: Abs: Coeficiente de absorción (\%)

$$
\text { Ps: Peso desecado (g) }
$$$$
\text { Pd: Peso saturado (g) }
$$

Con respecto al comportamiento de los pañetes aplicados sobre diferentes materiales, es importante destacar que en todos los casos su adherencia resultó adecuada, a excepción de la madera sobre la que se desprendieron tanto el mortero mezclado 
Tabla 1. Coeficiente de absorción

\begin{tabular}{|l|l|c|c|c|c|}
\hline Muestra & \multicolumn{1}{|c|}{ Mezcla } & $\begin{array}{c}\text { Peso desecado } \\
(\mathbf{g})\end{array}$ & $\begin{array}{c}\text { Peso saturado } \\
(\mathbf{g})\end{array}$ & $\begin{array}{c}\text { Agua absorbida } \\
(\mathbf{g})\end{array}$ & $\begin{array}{c}\text { Coeficiente de absorción } \\
(\mathbf{\%})\end{array}$ \\
\hline A & Tierra-arena-mucílago & 43,9 & 45,5 & 1,6 & $3,64 \%$ \\
\hline B & Tierra-arena-agua & 46,3 & 51,2 & 4.9 & $10,58 \%$ \\
\hline
\end{tabular}

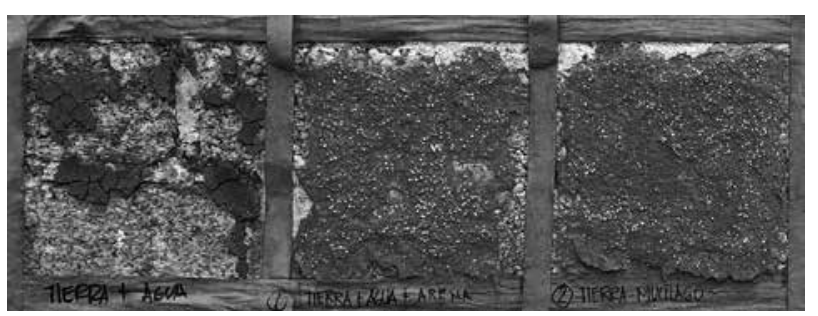

Figura 7. Se observa a la izquierda la pérdida de la muestra sin mucílago ni arena, en medio con arena y agua y la tercera con arena y mucílago. Fuente: elaboración propia.

con agua como el de mucílago. La colocación en todos los casos se inició a mano y posteriormente se alisó con un badilejo (palustre) con lo que se consiguieron superficies notablemente lisas y uniformes.

Finalmente, se han dejado a la intemperie por más de un año los recubrimientos realizados con agua y con soluciones con mucílago. En estas muestras, después de la intensa temporada de lluvias del 2018 el recubrimiento mezclado con agua tuvo una pérdida cercana al $80 \%$ mientras que el realizado con mucílago se mantiene en mejor estado. Ha perdido parte de su capa externa pero su volumen permanece con sorprendente integridad (Figura 6).

\section{Conclusiones}

Como resultado de los ensayos realizados a las diferentes mezclas se pudo poner en evidencia que el mucílago de nopal efectivamente modifica el comportamiento físico de las mezclas de tierra utilizadas para revoques. El hecho de que la longitud de las fracciones en las que se rompen las barras que fueron probadas haya pasado de $5,8 \mathrm{~cm}$ a $7,1 \mathrm{~cm}$ se vincula con un incremento en la cohesividad de las mezclas del orden del 22,4\%. Este dato lógicamente constituye un mejoramiento en su proceso de mezclado, aplicación, secado y permanencia en los muros.

Las combinaciones con el mucílago elevan considerablemente la trabajabilidad del material al posibilitar la realización de probetas sumamente delgadas que conservan su geometría y que presentan superficies notablemente lisas y homogéneas en ambas caras. Esta condición garantiza que los recubrimientos realizados con esta mezcla tendrán una superficie lisa y uniforme, que se traduce en mejor calidad visual, durabilidad e higiene.

Con respecto al proceso de hidratación, se comprobó que -para obtener condiciones similares de trabajabilidad del material al emplear mucílago-, se requiere 22,85\% menos de líquido que cuando se mezcla con agua. Esto significa que, al agregar mucílago al agua, se ahorrará el consumo de este vital líquido, lo que se traduce en un destacable beneficio ecológico.

Esta información cobra gran relevancia desde la perspectiva de la sostenibilidad ecológica. La tierra es un material que se caracteriza por un óptimo aprovechamiento del agua con respecto a los materiales convencionales de construcción, ya que utiliza comparativamente muy poca $y$, además, no la contamina. Pero si adicionalmente a esta cualidad general se consigue disminuir la cantidad de agua de mezclado, se potencializa la sostenibilidad del proceso.

Por otra parte, esta condición genera ventajas desde el punto de vista constructivo. Como se sabe, uno de los principales problemas de trabajar con tierra de alta o media plasticidad se deriva de su retracción al secado, la cual se manifiesta en agrietamientos y desprendimientos. En cambio, si se utiliza una menor cantidad de líquido de mezcla se tendrá un componente que conservará sus dimensiones, densidad e integridad al secar. 
Las experiencias asociadas a la velocidad en la pérdida de agua durante el endurecimiento de las probetas también resultaron muy positivas. El hecho de que las muestras que fueron amasadas con mucílago tardaran 9,2 horas en secarse mientras que las de agua lo hicieron en 6,33 horas representa un retardo en el tiempo de secado del $38 \%$. Esto se traduce en la conformación de un material más consistente y por lo tanto más durable ante el impacto la erosión y del agua, como se pudo demostrar con el resto de los experimentos.

Con los resultados obtenidos en las pruebas a compresión, se pudo determinar que las resistencias más altas se consiguen con la tierra mezclada con agua y que en cambio el mucílago redujo su resistencia. Una hipótesis sobre este resultado se vincula con el segundo aspecto analizado. Es posible que las probetas que fueron sometidas a compresión después de un mes todavía no hayan alcanzado su secado total aunque a simple vista parecieran secas.

Pero también puede ser que durante el llenado de los moldes, la presencia del mucílago pudiera haber generado un efecto de "rebote" en el proceso de compactación de las capas. Esta condición se traduce entonces en bloques con una menor densidad y por consiguiente su capacidad de carga disminuye. Será necesario hacer más pruebas al respecto para verificar si este resultado es constante.

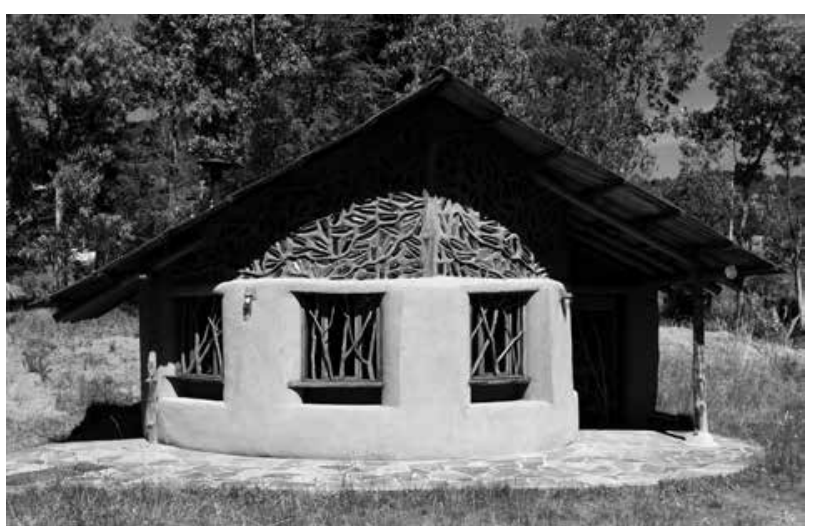

Figura 8. Pañetes de tierra expuestos a la intemperie en Tlaxco, Tlaxcala, México. Diseño de la Arquitecta Alejandra Caballero, Proyecto San Isidro. Fuente: Guerrero (2015).
Sin embargo, si se confirmara la segunda hipótesis podría pensarse que para el caso de los pañetes resulta positivo que tengan una menor densidad. Un revestimiento menos denso propicia un adecuado flujo de vapor de agua con el entorno, y por lo tanto, se mejora el comportamiento higrotérmico del sistema. Además, esta menor capacidad de carga también permite suponer un comportamiento más dúctil ante vibraciones y deformaciones como las que se presentan por ejemplo con hundimientos diferenciales, vibraciones y sismos.

En quinto lugar, con los ensayos de absorción capilar se confirmó que el material con mucílago no pierde su porosidad, pero sí disminuye radicalmente la velocidad de flujo del agua en estado líquido. El mucílago evita que pase alrededor del $300 \%$ de agua durante los procesos de absorción capilar, en el transcurso de los primeros minutos del proceso. La muestra estabilizada disminuyó su coeficiente de absorción de $10.58 \%$ a 3.64\%.

Finalmente, la observación de la posibilidad del empleo de pañetes de tierra estabilizada con mucílago no solamente sobre estructuras térreas sino también sobre superficies de cemento y ladrillo, abre un amplio abanico de opciones para mejorar su imagen, pero sobre todo, para el acondicionamiento bioclimático de los espacios.

Los componentes constructivos de tierra favorecen el equilibrio de la temperatura interior de los ambientes gracias a su masa e inercia térmica, así como el aislamiento derivado de la presencia de poros (Garzón y Neves, 2007). Asimismo, desarrollan un elevado potencial para acondicionar la absorción y desprendimiento de vapor de agua, proceso que incide directamente en la sensación higrotérmica de confort (Minke, 2005).

Se espera que paulatinamente las sociedades se vayan dando cuenta de las bondades del uso de la tierra como material constructivo desde la perspectiva de la sostenibilidad económica, ecológica y socio cultural. En ese proceso de sensibilización, el uso de pañetes de tierra puede ser fundamental pues, además de mejorar las condiciones de habitabilidad de los espacios, progresivamente hará 
que los habitantes reconozcan a la tierra como parte de su vida cotidiana.

Uno de los problemas actuales de las ciudades con respecto al olvido de los sistemas constructivos tradicionales radica en el simple hecho de que la gente no los ve. Dar visibilidad a la tierra permitirá recuperar su protagonismo social y propiciará que las comunidades retomen el camino de la independencia tecnológica y cultural que representa la posibilidad de realizar y mejorar con las propias manos el lugar en el que viven, utilizando el material más ecológico que existe (Figura 8).

\section{Referencias bibliográficas}

\section{ASTM (2006). C 39/C 39M. Standardized} test method for resistance to compression of cylindrical concrete specimens. USA: ASTM International.

Cruz, S. (2013). El mucílago de nopal como aditivo de las pastas de cal empleadas en conservación. En Barba, L. \& Villaseñor, I. (Eds.). La cal: Historia, propiedades y usos. México: IIAUNAM y ANFACAL, A.C., pp. 183-202.

Garzón, L. y Neves, C. (2007). Investigar, formar, capacitar, transferir. Los grandes desafios de la arquitectura y construcción con tierra. Apuntes, 20 (2), pp. 324-335.

Guerrero, L., Correia, M. y Guillaud, H. (2012). Conservación del patrimonio arqueológico construido con tierra en Iberoamérica. Apuntes, 25 (2), pp. $210-225$.

Guerrero, L. (2015). Sostenibilidad y conservación del patrimonio edificado. Revista Palapa, III, 1 [17], pp. 73-84.

Guerrero, L. (2016). El papel de la humedad y la compactación en la elaboración de recubrimientos de tierra. Revista Construcción con tierra CT7, pp. 11-22.

Juárez, E. y Rico, A. (2010). Mecánica de suelos. Tomo I, Fundamentos de la mecánica de suelos. México D.F., México: Limusa.
Kita, Y. y Daneels, A. (2015). Evaluación de bitumen como estabilizante para patrimonio construido en tierra bajo el clima trópico húmedo. Estudios sobre conservación, restauración y museografía, 2, pp. 129-144.

Lozano, A. (2008). La piel del edificio. Cali, Colombia: Universidad del Valle.

Minke, G. (2005). Manual de construcción en tierra. Montevideo, Uruguay: Fin de Siglo.

NMX-C-036-ONNCCE (2004). Industria de la construcción. Bloques, tabiques o ladrillos, tabicones y adoquines -resistencia a la compresión. Método de prueba. México D.F., México: Organismo Nacional de Normalización y Certificación de la Construcción y la Edificación, S.C.

Normal 11/85 (1985). Assorbimento d'acqua per capillarità-Coeficiente di assorbimento capillare. Italia: CNR-ICR.

Pérez, N. (2009). Formulación de un mortero de inyección con mucílago de nopal para restauración de pintura mural. Tesis para obtener el Grado de Ingeniero Químico. Guadalajara, México: ITESO.

Pérez, A.; Guerrero, L.; González, J. y Prado, R. (2017). Nopal mucilage as hydration agent for quicklime; extraction methods. Ge-conservación, 11, pp. 189-195.

Torres, P.; Cruz, S., Peña, N., Fernández, S. E., Rodríguez, M. y Cruz, A. (2015). La baba y el mucílago de nopal, una alternativa natural para la conservación de acabados arquitectónicos de tierra. Revista Interdisciplinaria, INAH, 99, pp. 93-114.

Ramírez-Arellanes, S., Cano-Barita, P. F. de J., Julián-Caballero, F. y Gómez-Yañez, C. (2012). Propiedades de durabilidad en hormigón y análisis microestructural en pastas de cemento con adición de mucílago de nopal como aditivo natural. Materiales de Construcción, 62, 307, pp. 327-341.

Warren J. (1999). Conservation of earth structures. Oxford, U.K.: Butterworth Heinemann. 